\title{
Functionalization of silicon nanowires with transition metal atoms
}

\author{
E. Durgun, ${ }^{1}$ N. Akman, ${ }^{2,3}$ and S. Ciraci ${ }^{1,3, *}$ \\ ${ }^{1}$ Department of Physics, Bilkent University, Ankara 06800, Turkey \\ ${ }^{2}$ Department of Physics, Mersin University, Mersin 33343, Turkey \\ ${ }^{3}$ UNAM-Institute of Materials Science and Nanotechnology, Bilkent University, Ankara 06800, Turkey \\ (Received 7 May 2008; revised manuscript received 23 September 2008; published 18 November 2008)
}

\begin{abstract}
This paper investigates atomic structure, mechanical, electronic, and magnetic properties of silicon nanowires ( $\mathrm{SiNW}$ ) using first-principles plane-wave calculations within density-functional theory. We considered bare, hydrogen-terminated, and 3d-transition metal (TM) adsorbed SiNWs oriented along [001] direction. We also studied Cr interstitial impurity. Nanowires of different sizes are initially cut from the bulk Si crystal in rodlike forms, and subsequently their atomic structures are relaxed before and also after the termination of surface dangling bonds by hydrogen atoms. We first presented an extensive analysis of the atomic structure, stability, elastic, and electronic properties of bare and hydrogen-terminated SiNWs. The energetics of adsorption and resulting electronic and magnetic properties are examined for different levels of $3 d-\mathrm{TM}$ atom coverage. Adsorption of TM atoms generally results in magnetic ground state. The net magnetic moment increases with increasing coverage. While specific SiNWs acquire half-metallic behavior at low coverage, at high coverage ferromagnetic nanowires become metallic for both spin directions, and some of them have very high spin polarization at the Fermi level. Our results suggest that the electronic and spintronic devices with conducting interconnects between them can be fabricated on a single SiNW at a desired order. We believe that our study will initiate new research on spintronic applications of SiNWs.
\end{abstract}

DOI: 10.1103/PhysRevB.78.195116

PACS number(s): 72.25. $-\mathrm{b}, 73.63 . \mathrm{Nm}, 75.75 .+\mathrm{a}$

\section{INTRODUCTION}

One of the major obstacles in miniaturization of solidstate electronic devices has been the fabrication of interconnects having diameters compatible with the size of devices they connect. Not only in solid-state electronics but also in nanoelectronics, interconnecting nanodevices or molecular devices has remained a challenge for several decades. An active research was carried out during the last decade on the mechanical, electronic, and transport properties of onedimensional (1D) nanowires, including monoatomic chains, wires having a few atoms in their cross section, ${ }^{1,2}$ and nanotubes. ${ }^{3}$ Initially, suspended monoatomic chains of gold has been a breakthrough in the field. ${ }^{4}$ Presently, the practical applications of monoatomic chains appear to be unrealistic even if their stability and quantum transport properties have been understood to a great extent. Recently carbon nanotubes have been the focus of attention owing to their nanoscale diameter and regular cylindrical shape. Furthermore, the outer surface of these tubes can be coated uniformly by $\mathrm{Ti}$ atoms allowing high conductance. ${ }^{5,6}$ Despite these advantages, the use of carbon nanotubes as an interconnect are hindered because of difficulties in controlling their diameter during the synthesis.

While the search for feasible interconnects in nanoelectronics are continuing, nanosized Si nanowires (SiNW) have appeared to be an attractive 1D material because of the wellknown silicon-based microelectronic fabrication technology and their use directly on the Si-based chips. SiNWs display diversity of electronic properties depending on the number of $\mathrm{Si}$ atoms in their cross section. The linear monatomic $\mathrm{Si}$ chain is metallic with quantum ballistic conductance three times higher than that of gold. ${ }^{7}$ The linear Si chain is, however, metastable and hence it changes to a metallic zigzag chain. ${ }^{7}$ Stable nanowires made of Si pentagons perpendicular to the wire axis have been reported. ${ }^{8}$ Based on the prediction of first-principles calculations, Si can also form tubular structures made of hexagons similar to that of carbon nanotubes. Depending on the chirality and diameter, these tubular structures can exhibit metallic or semiconducting behaviors. ${ }^{9}$

Silicon nanowires have been produced using various experimental methods. ${ }^{10-14}$ The formation of an oxide layer around SiNW with a minimum thickness of $1-3 \mathrm{~nm}$ appears to be a disadvantage. ${ }^{15}$ Nevertheless, rodlike SiNWs with a diameter of 1.3-7 $\mathrm{nm}$ have been prepared more recently. Their oxide layer can be removed from their surface and the dangling bonds at the surfaces can be terminated with hydrogen. ${ }^{16}$ The experimental findings have indicated a crystalline core surrounded by an outer surface for the atomic structure of the SiNWs. Whether this crystalline core is tetrahedral (diamondlike as in bulk $\mathrm{Si}$ ) or is of any other arrangement of atoms has been the subject of further investigation. Fullerene-like (cagelike) nanowires have been proposed and investigated theoretically in Refs. 17 and 18 . Diamondlike and cagelike wires are compared energetically in Ref. 18. Besides, single crystalline and polycrystalline natures of very thin silicon nanowires have been theoretically investigated by Zhao and Yakobson. ${ }^{19}$

The thinnest SiNW (having radius $R \sim 0.65-3.5 \mathrm{~nm}$ ) synthesized so far has been grown along the [110] and the [112] directions. ${ }^{16}$ SiNWs with sizes less than $10 \mathrm{~nm}$ have been also reported along different directions such as [001], ${ }^{20}$ $[110],{ }^{20,21}[111],{ }^{21}$ and [112]. ${ }^{21}$ Theoretically [001] oriented SiNW is the most studied wire in the literature. Rurali and Lorente ${ }^{22}$ studied nonpassivated and undoped [001] SiNWs consisting of 57, 114, and 171 atoms by using densityfunctional theory (DFT). They obtained energetically equivalent metallic and semimetallic reconstructions. Tight-binding density-functional calculations on hydrogen-passivated 
SiNWs (Ref. 23) have presented that, while [112] wires are the most stable ones, [001] wires have the widest energyband gap. By passivating the SiNW surface with some halogens including $\mathrm{Br}, \mathrm{Cl}$, and $\mathrm{I}$, the electronic structure of different sized wires ranging from 0.6 to $3 \mathrm{~nm}$ have been studied using $a b$ initio density-functional theory calculations. $^{24}$

Experimental and theoretical studies so far have shown that those SiNWs exhibit a wide range of physical and chemical properties, which may be of interest in technological applications. ${ }^{25,26}$ Depending on whether the dangling bonds on their outer surface are saturated with hydrogen atoms and the shape of their cross section, they exhibit semiconducting or metallic behavior. Usually, unsaturated dangling bonds on the outer surface attribute a metallic character to a SiNW. Upon saturation of these dangling bonds, a SiNW becomes a semiconductor. Moreover, the band gap of semiconductor SiNWs vary with their diameter. Not only the band gap but also the stability of a SiNW strongly depends on its diameter.

Silicon nanowires can function in various electronic and optical applications such as field effect transistors ${ }^{27}$ (FETs), light emitting diodes, ${ }^{28}$ lasers, ${ }^{29}$ and interconnects. Its conductance can be tuned by doping during the production process or by applying a gate voltage in a SiNW FET. In various device applications, such as diodes and transistors, $n$ - and p-doped SiNWs (Refs. 25, 30, and 31) have also attracted interest. Furthermore a growing research interest has been devoted to the functionalization of SiNW surface with various species to study the chemical and biological sensitivities of silicon nanowires. ${ }^{32-34} \mathrm{H}$-saturated SiNW and GeNW, which have band gaps of different widths, can form pseudomorphic heterostructures. ${ }^{35}$ It has been predicted that the periodic, H-saturated $\operatorname{SiNW}(N) / \operatorname{GeNW}(N)$ heterostructure superlattices behave as a multiple quantum well structure with confined states. ${ }^{35}$

This paper presents an extensive study on the mechanical, electronic, and magnetic properties of bare, H-passivated SiNWs oriented along the [001] direction, as well as those functionalized by $3 d$-transition metals (TMs). Preliminary results of this study were published as a short letter. ${ }^{36}$ Our study is based on the first-principles plane-wave calculations using DFT and ab initio molecular-dynamics (MD) calculations using microcanonical ensemble. The organization of the paper is as follows: in Sec. II, we summarize essential aspects of the method and its relevant parameters used in the calculations. In Sec. III we present a systematic and brief summary of our results on the atomic structure, reconstruction, energetics, mechanical, and electronic properties of bare and hydrogen-terminated SiNWs of different cross sections. Section IV deals with the functionalization of SiNWs with light TMs, such as Ti, Fe, Co, Cr, and Mn. First, adsorption geometry and energetics are examined for the adsorption of a single TM atom (per primitive cell designated as $\Theta=1$ ) on the surface of SiNWs. It was found that TM-doped $\mathrm{H}$-saturated SiNWs have ferromagnetic ground state and some of them exhibit half-metallic (HM) behavior with $100 \%$ spin polarization at the Fermi level $\left(E_{F}\right) \cdot{ }^{37,38}$ Upon increased coverage of TM atoms (corresponding to $\Theta=n$, with $n$ being the number of TM atoms adsorbed per primitive cell) of H-saturated SiNWs, the HM character is disturbed but spin polarization continues to be very high for specific nanowires. High magnetic moment and also high spin polarization for certain cases achieved at high $\Theta$ can be important for applications in biotechnology and spintronics. Our results are of fundamental and technological interests since roomtemperature ferromagnetism is already discovered in $\mathrm{Mn}^{+}$-doped SiNW. ${ }^{39}$ The paper is concluded with our conclusions in Sec. V.

\section{METHOD}

We have performed first-principles plane-wave calculations $^{40-42}$ within DFT. ${ }^{43}$ All calculations for nonmagnetic, ferromagnetic, and antiferromagnetic states are carried out using ultrasoft pseudopotentials. ${ }^{41,44}$ The results of these calculations were also confirmed by calculations using projector augmented wave (PAW) potential. ${ }^{45}$ The exchangecorrelation potential has been approximated by generalized gradient approximation (GGA) (Ref. 46) both for spinpolarized and spin-unpolarized cases. For partial occupancies we used the Methfessel-Paxton smearing method. ${ }^{47}$ All structures have been treated within supercell geometry using the periodic boundary conditions with cell dimensions of $a$ and $b$ ranging from 20 to $30 \AA$, depending on the diameter of the SiNW, in order to provide $10 \AA$ vacuum space, and along the wire axis $c=c_{0}\left(c_{0}\right.$ being the relaxed lattice constant of $\mathrm{SiNW}$ ). In the self-consistent potential and total-energy calculations, the Brillouin zone (BZ) of supercell is sampled in the $\mathbf{k}$ space within Monkhorst-Pack scheme ${ }^{48}$ by $(1 \times 1$ $\times 15)$ mesh points. A plane-wave basis set with maximum kinetic energy of $350 \mathrm{eV}$ has been used.

For the calculation of magnetic moments, the charge density of spin-up and spin-down electrons is calculated separately. Spin-polarized total energy $E_{T}^{\mathrm{sp}}$ is calculated by starting with different initial magnetic-moment $(\mu)$ values and subsequently by relaxing them together with the atomic structure. This way, the trapping of the magnetic moment in a local minimum has been avoided. To check whether antiferromagnetic ground state of $\mathrm{H}$-saturated $\operatorname{SiNW}(N)+\mathrm{TM}$ exists, spin-polarized calculations have been carried out in supercell including double primitive cell of the nanowire, where adjacent TM atoms may have opposite spins.

All atomic positions and lattice constants are optimized by using the conjugate gradient method where total energy and atomic forces are minimized. The convergence for energy is chosen as $10^{-6} \mathrm{eV}$ between two ionic steps, and the maximum force allowed on each atom is $10^{-3} \mathrm{eV} / \AA$. To relieve the constraints imposed by periodic boundary conditions, the calculations related with the study of reconstruction have been repeated using supercells with $c=2 c_{0}$ and $4 c_{0}$ (namely, comprising double and quadruple unit cells of SiNW, respectively).

\section{PROPERTIES OF BARE AND HYDROGEN- PASSIVATED SILICON NANOWIRES}

In this paper, we consider the rodlike bare and H-terminated Si nanowires oriented along [001] direction 

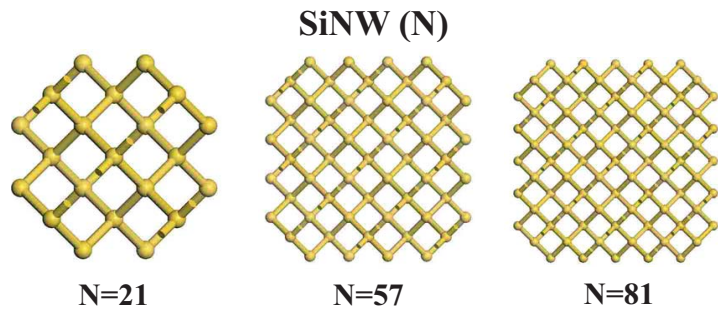

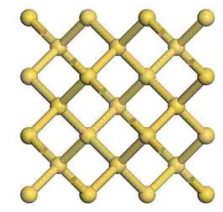

$\mathbf{N}=\mathbf{2 5}$

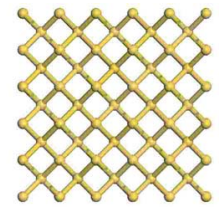

$\mathbf{N}=61$
FIG. 1. (Color online) Atomic structure of the cross section of rodlike Si nanowires as cut from the ideal bulk silicon [i.e., ideal $\operatorname{SiNW}(N)]$ for $N=21,25,57,61$, and 81 .

having different diameters (or different number of $\mathrm{Si}$ atoms in their unit cell). The bare Si nanowire oriented along [001] direction and having $N \mathrm{Si}$ atoms in the primitive unit cell is specified by $\operatorname{SiNW}(N)$. Similarly, a nanowire with all dangling bonds on the surface passivated by $\mathrm{H}$ atoms after relaxation is specified by $\mathrm{H}-\mathrm{SiNW}(N)$. If the dangling bonds are saturated before relaxation it is specified as $\mathrm{H}-\mathrm{SiNW}(N)-b$. The sequence of structure optimization is crucial for the electronic properties. Here, the structure optimization of H-SiNWs is achieved in two steps. Initially, SiNWs are cut in rodlike forms from the bulk Si crystal (see Fig. 1). Subsequently, the initial atomic structure having ideal bulk configuration is relaxed to yield the minimum total energy with the atomic forces smaller than $10^{-3} \mathrm{eV} / \AA$ (see upper panels of Fig. 2). In the second step, hydrogen passivation of bare SiNWs is achieved by termination of the dangling bonds of $\mathrm{Si}$ atoms (which have nearest-neighbor coor-

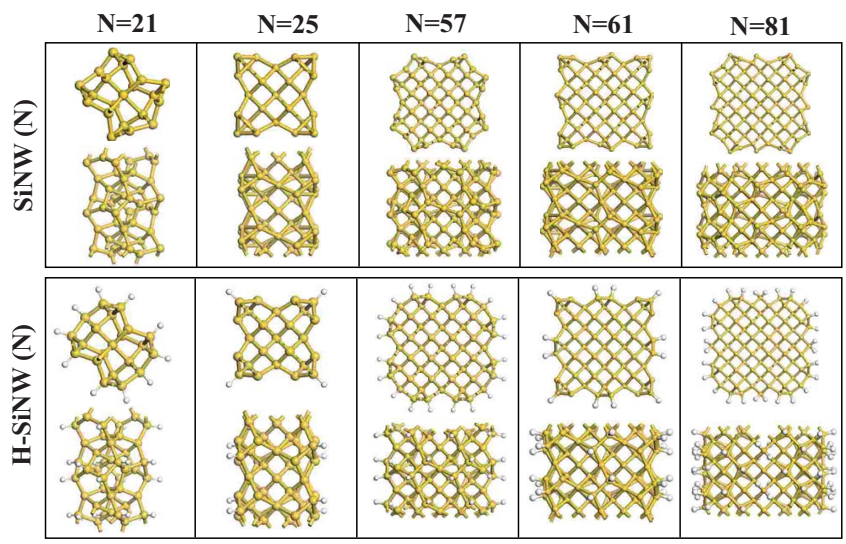

FIG. 2. (Color online) Upper panels: Top and side views of optimized atomic structures of bare $\operatorname{SiNW}(N)$ s (with $N=21,25,57$, 61, and 81) cut from the bulk Si crystal along [001] direction. Lower panels: $\mathrm{H}-\mathrm{SiNW}(N)$ s which are optimized upon $\mathrm{H}$ termination of $\operatorname{SiNW}(N)$ s in the upper panel. Large and small balls indicate $\mathrm{Si}$ and $\mathrm{H}$ atoms, respectively. Side views consist of two primitive unit cells. dination less than four $\mathrm{Si}$ atoms) by $\mathrm{H}$ atoms. Subsequently, whole structure that includes $\mathrm{H}$ atoms is optimized again (see lower panels of Fig. 2). An alternative way of structure optimization was also carried out in several theoretical studies in single step: first, the dangling bonds on the surface of the ideal bare SiNW cut from the bulk crystal are saturated by $\mathrm{H}$ atoms and subsequently whole structure has been optimized once. The latter optimization process, however, leads to electronic structure different from the former two-step process done in the present study. It should be noted that the two-step process better mimics the actual growth process. The results for single-step relaxation, i.e., H-SiNW $(N)-b$, are also included for comparison. This section presents a comparative study of atomic structure, reconstruction, electronic band structure, and elastic properties of structure-optimized bare and H-passivated SiNWs. Thereafter, it will be clear that the properties of optimized bare SiNWs are quite different from those of H-SiNWs.

\section{A. Atomic structure and energetics}

Figure 2 shows the atomic structures of optimized bare SiNWs and $\mathrm{H}-\mathrm{SiNW}$ investigated in this paper. We considered wire geometries having 21, 25, 57, 61, and $81 \mathrm{Si}$ atoms in the primitive unit cell. Among these wires, nanowires 21, 57 , and 81 have rather round cross sections while those of 25 and 61 have squarelike cross sections.

The cohesive energy (per Si atom) of the bare SiNWs is calculated by $\bar{E}_{c}=E_{T}[\mathrm{Si}]-E_{T}[\mathrm{SiNW}(N)] / N$, in terms of the total energy of free $\mathrm{Si}$ atom and the total energy of bare $\operatorname{SiNW}(N)$ having optimized structure. According to this definition, $\bar{E}_{c}>0$ indicates that the structure is stable with respect to constituent free atoms. The cohesive energy relative to the bulk Si crystal, $\bar{E}_{c}^{\prime}=E_{T}[\mathrm{Bulk} \mathrm{Si}]-E_{T}[\operatorname{SiNW}(N)] / N$, is calculated by replacing the free-atom energy with the total energy of bulk equilibrium crystal per $\mathrm{Si}$ atom, i.e., $E_{T}$ [Bulk Si]. The binding energy of hydrogen with respect to the free $\mathrm{H}$ atom is also of interest and is calculated by $E_{b}$ $=\left(N_{\mathrm{H}} E_{T}[\mathrm{H}]+E_{T}[\operatorname{SiNW}(N)]-E_{T}[\mathrm{H}-\mathrm{SiNW}]\right) / N_{\mathrm{H}}$, in terms of the total energy of structure-optimized bare $\operatorname{SiNW}(N)$ and $\mathrm{H}-\mathrm{SiNW}(N)$ terminated by $N_{\mathrm{H}}$ hydrogen atom and the energy of free $\mathrm{H}$ atom, $E_{T}[\mathrm{H}]$. The binding energy of hydrogen relative to $\mathrm{H}_{2}$ molecule, $E_{b}^{\prime}$, is obtained if $E_{T}[\mathrm{H}]$ is replaced by $E_{T}\left[\mathrm{H}_{2}\right] / 2$. Calculated structure parameters, such as the lattice constant along the wire axis $c_{0}$, maximum radius of the cross section, cohesive energy relative to free $\mathrm{Si}$ atom and Si crystal, and binding energy of $\mathrm{H}$ atom, are presented in Tables I and II.

In general, the lattice constant $c_{0}$ of optimized bare and $\mathrm{H}-\mathrm{SiNW}(N)$ decreases with increasing $N$. Namely, the lattice constant is large for small $N$ but approaches the lattice constant of bulk Si. This trend is, however, different for $N=25$ and 61 , which form a class of nanowires with squarelike cross sections.

$\bar{E}_{c}$ is comparable with the cohesive energy of bulk crystal calculated to be $4.46 \mathrm{eV} / \mathrm{atom}$, and expectantly it increases with increasing $N$. As for the average cohesive energy relative to the bulk $\mathrm{Si}$ crystal $\bar{E}_{c}^{\prime}$, it is small but negative as 
TABLE I. Lattice constant $c_{0}$, maximum radius $R_{\max }$, cohesive energy per atom relative to free $\mathrm{Si}$ atom $E_{c}$, cohesive energy per atom relative to crystalline $\mathrm{Si}$, minimum of the band gap $E_{G}(\mathrm{M}=$ metal), and elastic constant $\kappa$ of bare $\operatorname{SiNW}(N)$.

\begin{tabular}{lcccccc}
\hline \hline $\operatorname{SiNW}(N)$ & $\begin{array}{c}c_{0} \\
(\AA)\end{array}$ & $\begin{array}{c}R_{\max } \\
(\AA)\end{array}$ & $\begin{array}{c}\bar{E}_{c} \\
(\mathrm{eV})\end{array}$ & $\begin{array}{c}\bar{E}_{c}^{\prime} \\
(\mathrm{eV})\end{array}$ & $\begin{array}{c}E_{G} \\
(\mathrm{eV})\end{array}$ & $\begin{array}{c}\kappa \\
\left(\mathrm{eV} / \AA^{2}\right)\end{array}$ \\
\hline$N=21$ & 5.68 & 8.31 & 4.05 & -0.59 & 0.6 & 133 \\
$N=25$ & 5.33 & 10.76 & 4.13 & -0.51 & $\mathrm{M}$ & 161 \\
$N=57$ & 5.42 & 15.59 & 4.24 & -0.40 & $\mathrm{M}$ & 339 \\
$N=61$ & 5.38 & 18.43 & 4.26 & -0.38 & $\mathrm{M}$ & 375 \\
$N=81$ & 5.40 & 18.73 & 4.27 & -0.37 & $\mathrm{M}$ & 432 \\
\hline \hline
\end{tabular}

expected. However, its magnitude becomes smaller as $N$ increases (namely as the bulk properties start to dominate with increasing diameter). The binding of $\mathrm{H}$ atom is exothermic with positive binding energy both for $E_{b}$ and also for $E_{b}^{\prime}$.

Atomic structure of SiNWs was analyzed by comparing the distribution of interatomic distances between various atoms (up to sixth nearest neighbor) with that of ideal crystal. In Fig. 3, the distribution of interatomic distances are plotted for bare $\operatorname{SiNW}(N), \mathrm{H}-\operatorname{SiNW}(N)$, as well as $\mathrm{H}-\operatorname{SiNW}(N)-b$ for $N=21,57$, and 81. Similar analysis is illustrated in Fig. 4 for $N=25$ and 61, which have rather square cross sections.

The deviations from ideal case are large for small $N$. The distribution of the first nearest-neighbor distance is always sharp for all $N$. Significant deviations from the second-, third-, etc. nearest-neighbor distances of ideal bulk crystal are observed. In particular, already the peak related to the distribution of second-nearest-neighbor distance starts to broaden. The structure of optimized bare SiNWs is healed by $\mathrm{H}$ termination of dangling bonds. This is seen by the fact that the distribution of distant nearest neighbors appears as peaks coinciding with the ideal structure. Additional peaks are related with surface reconstruction. Clearly, the distribution of $\mathrm{H}-\mathrm{SiNW}(N)-b$ is very similar to that of ideal SiNW since the surface relaxation is hindered by saturating the dangling bonds of ideal SiNW.

\section{B. Reconstruction and stability}

The surface reconstruction of bare SiNWs is crucial with the resulting electronic properties and hence has been widely discussed. ${ }^{19,49-51}$ To ensure that the structures discussed in Fig. 2 are minimum-energy structure, we performed structure optimization by doubling and also by quadrupling the unitcell size, where the lattice constant of the supercell is taken to be $c=2 c_{0}$ and $4 c_{0}$, respectively. As initial structures, we took always the structure of ideal SiNW as cut from the ideal bulk crystal. All efforts to optimize the atomic structure of SiNWs resulted with the atomic structures presented in Fig. 2. All the surface atoms prefer to dimerize in order to minimize the total energy. Our results are in agreement with the studies considering the reconstruction patterns for the SiNWs in the same [001] growth direction. ${ }^{19,49-51} \mathrm{Cao}$ et al. ${ }^{50}$ even labeled $N=25$ and 61 as magic numbers since they give the lowest energy dips in the plot of formation energies versus $N$. We also obtained the same geometry with Rurali et al. ${ }^{51}$ for $N=21,57$, and 81 for [001] SiNWs with (110) facets which confirms the results.

Interestingly, we found a second type of reconstruction for $\operatorname{SiNW}(21)$ for double cell geometry. $E_{T}$ of second system is found to be $0.54 \mathrm{eV} /$ cell higher in energy but stable. The corresponding $E_{C}$ is calculated as $4.03 \mathrm{eV} /$ atom. This structure is also semiconducting with $E_{G}=0.30 \mathrm{eV}$. When we saturate all dangling bonds on the surface, $E_{G}$ becomes 2.0 $\mathrm{eV}$. Since the radius of $\operatorname{SiNW}(21)$ is very small, the core cannot preserve the diamond structure. As a result, it leads to different structural and electronic properties.

We note that the global minimum for $\mathrm{Si}$ is the crystal in diamond structure; nanowires described above are only local minima in the Born-Oppenheimer surface. In this respect the stability of the wire structures presented in Fig. 2 is of prime importance. A thorough analysis of the stability of a SiNW is

TABLE II. Lattice constant $c_{0}$, maximum radius $R_{\max }$, binding energy of hydrogen relative to free $\mathrm{H}$ atom $E_{b}$, binding energy of hydrogen relative to $\mathrm{H}_{2}$ molecule $E_{b}^{\prime}$, minimum of the band gap $E_{G}$, and elastic constant $\kappa$ of hydrogen-terminated $\operatorname{SiNW}(N)$. The $\kappa$ values for hydrogen-terminated SiNWs before relaxation is given in parenthesis.

\begin{tabular}{lcccccc}
\hline \hline H-SiNW $(N)$ & $\begin{array}{c}c_{0} \\
(\AA)\end{array}$ & $\begin{array}{c}R_{\max } \\
(\AA)\end{array}$ & $\begin{array}{c}E_{b} \\
(\mathrm{eV})\end{array}$ & $\begin{array}{c}E_{b}^{\prime} \\
(\mathrm{eV})\end{array}$ & $E_{G}$ & $\begin{array}{c}\kappa \\
\left(\mathrm{eV} / \AA^{2}\right)\end{array}$ \\
\hline$N=21$ & 5.85 & 11.03 & 4.14 & 0.74 & 0.7 & $172(82)$ \\
$N=25$ & 5.45 & 13.20 & 4.22 & 0.82 & 1.1 & $254(96)$ \\
$N=57$ & 5.39 & 17.76 & 4.28 & 0.88 & 1.7 & $394(298)$ \\
$N=61$ & 5.43 & 20.90 & 4.34 & 0.94 & 1.1 & $509(303)$ \\
$N=81$ & 5.40 & 21.6 & 4.28 & 0.88 & 1.6 & $532(456)$ \\
\hline \hline
\end{tabular}



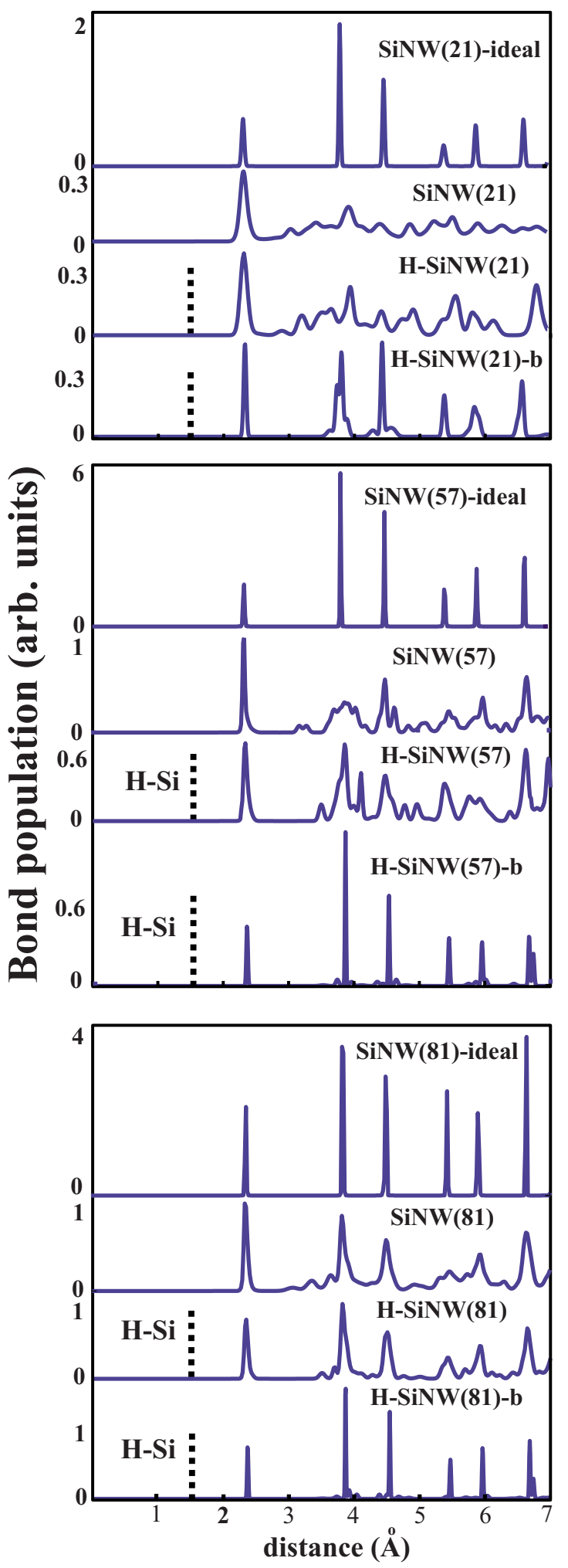

FIG. 3. (Color online) Upper curve in each panel with numerals indicates the distribution of first-, second-, third-, fourth-, etc., nearest-neighbor distances of the ideal $\operatorname{SiNW}(N)$ for $N=21,57$, and 81 as cut from the ideal $\mathrm{Si}$ crystal. The second curve is for the structure-optimized bare $\operatorname{SiNW}(N)$. The third curve is for the structure-optimized H-SiNW $(N)$. The bottom curve corresponds to $\mathrm{H}-\mathrm{SiNW}(N)-b$ (see text). Vertical dashed line corresponds to the distance of $\mathrm{Si}-\mathrm{H}$ bond.

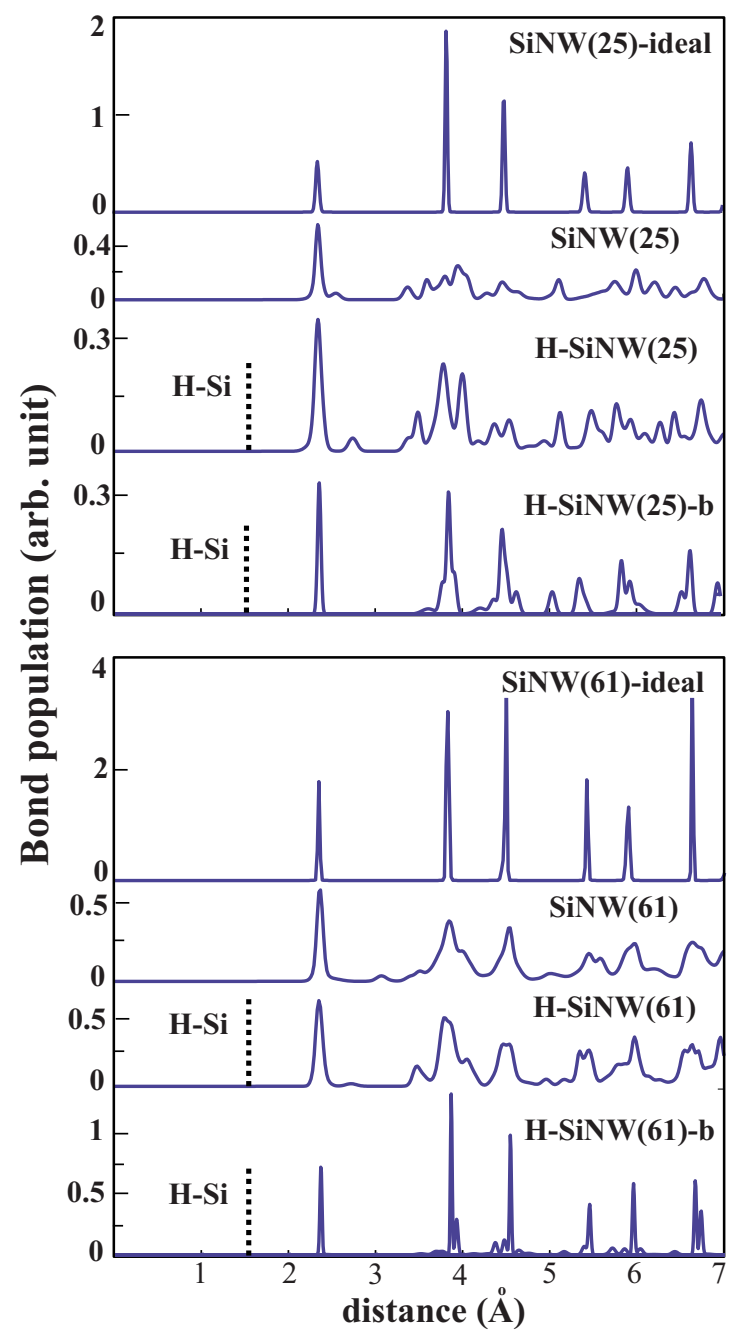

FIG. 4. (Color online) Upper curve in each panel with numerals indicates the distribution of first-, second-, third-, fourth-, etc., nearest-neighbor distances of ideal $\operatorname{SiNW}(N)$ for $N=25$ and 61 as cut from the ideal Si crystal. The second curve is for structureoptimized bare $\operatorname{SiNW}(N)$. The third curve is for the structureoptimized $\mathrm{H}-\mathrm{SiNW}(N)$. The bottom curve corresponds to $\mathrm{H}-\mathrm{SiNW}(N)-b$ (see text). Vertical dashed line corresponds to the distance of $\mathrm{Si}-\mathrm{H}$ bond.

necessary before one aims at its possible application as an electronic device. In addition to structural optimization using conjugate gradient method, the stability of structures have been tested by performing $a b$ initio MD calculations at 500 $\mathrm{K}$ for $1 \mathrm{ps}$ with $1 \mathrm{fs}$ time steps. In order to lift constraints imposed by single unit cell, MD calculations have been performed in a periodically repeating supercell with $c=4 c_{0}$.

\section{Elastic properties}

The elastic properties of a SiNW can be deduced by calculating its elongation under a uniaxial tensile stress along the axis of the wire. The response of the wire to a uniaxial tensile stress can be formulated by the expression $X_{x}=\kappa e_{x x}$, where $\kappa$ is Young's modulus and $e_{x x}$ is the strain along $x$ direction. $\kappa$ can be related to the elastic stiffness constants $C_{11}$ and $C_{12}$ in cubic crystals. However, since SiNWs studied 


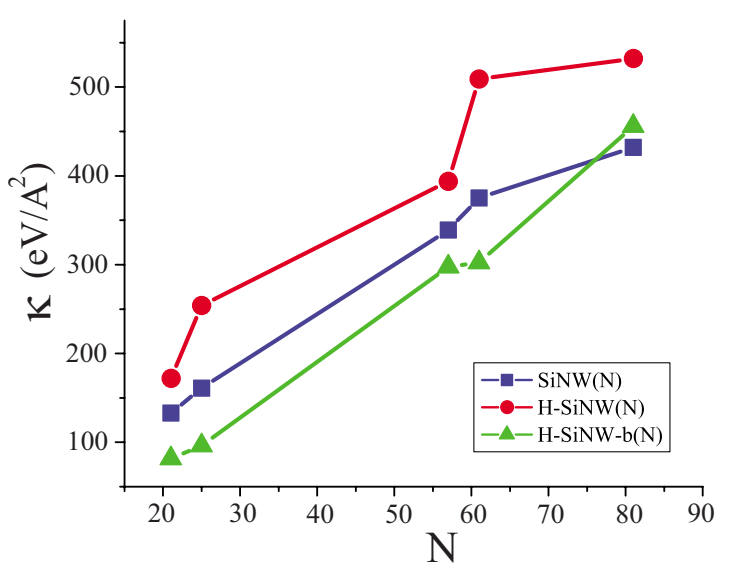

FIG. 5. (Color online) Variation in elastic strength $\kappa$ with number of atoms $N$ in the primitive unit cell of the optimized bare SiNWs, H-SiNW $(N)$ s, and $\mathrm{H}-\operatorname{SiNW}(N)-b$. Wires with $N=21,57$, and 81 have round cross sections, whereas wires with $N=25$ and 61 have squarelike cross sections.

here have high surface-to-volume ratio, it is better to define $\kappa$ directly from the relation, $\kappa=\partial E_{T}^{2} / \partial c_{0}^{2}$. To this end we calculated self-consistent total energy $E_{T}$ as a function on the lattice constant $c_{0}$ by relaxing all atomic positions under that constraint. The variation in $\kappa$ with $N$ is presented in Fig. 5 for bare and hydrogen-terminated SiNWs. One captures the following general trends from this figure: (i) The stiffness and also strength of a bare SiNW increases upon hydrogen termination of dangling bonds. (ii) The $\mathrm{H}-\operatorname{SiNW}(N)-b$ is the weakest among all types, indicating that surface reconstruction makes SiNWs stronger. (iii) For bare $\mathrm{SiNW} \kappa$ increases with $N$ almost linearly. $\operatorname{SiNW}(N)$ with $N=25$ and 61 shows slight deviation from the linear relation due perhaps to their squarelike cross sections. The deviation from the linearity is enhanced in the case of $\mathrm{H}-\operatorname{SiNW}(N)$ s. As compared to hydrogen-terminated SiNWs with round cross sections $(N$ $=21,57$, and 81), hydrogen-terminated wires with squarelike cross sections $(N=25$ and 61$)$ fall in a different category. It appears that upon $\mathrm{H}$ termination the strength of the latter wires increases more relative to the wires with round cross sections. As with the electronic structure, these results point to the fact that the mechanical properties are sensitive to the geometry of the SiNW at small diameter.

\section{Electronic energy structure}

Bare SiNWs are usually metallic due to the unsaturated bonds on the surface. For periodic structures these dangling bonds form surface bands, which occur in the band gap of the semiconductor and pin $E_{F}$. In this respect the danglingbond surface states are localized at the surface. In Figs. 6 and 7 the energy-band structures calculated for bare SiNWs confirm this argument except $\operatorname{SiNW}(21)$, which is semiconducting with band gap of $E_{G}=0.6 \mathrm{eV}$. Owing to the even number of dangling bonds, the related bands of $\operatorname{SiNW(21)}$ are filled. Moreover, due to the very small diameter of cross section, the dangling-bond surface states mix with bulklike bond (back bond) states. On the other hand, $\operatorname{SiNW}(N)$ for $N=25$, 57,61 , and 81 is metallic. Since these metallic wires have $n$

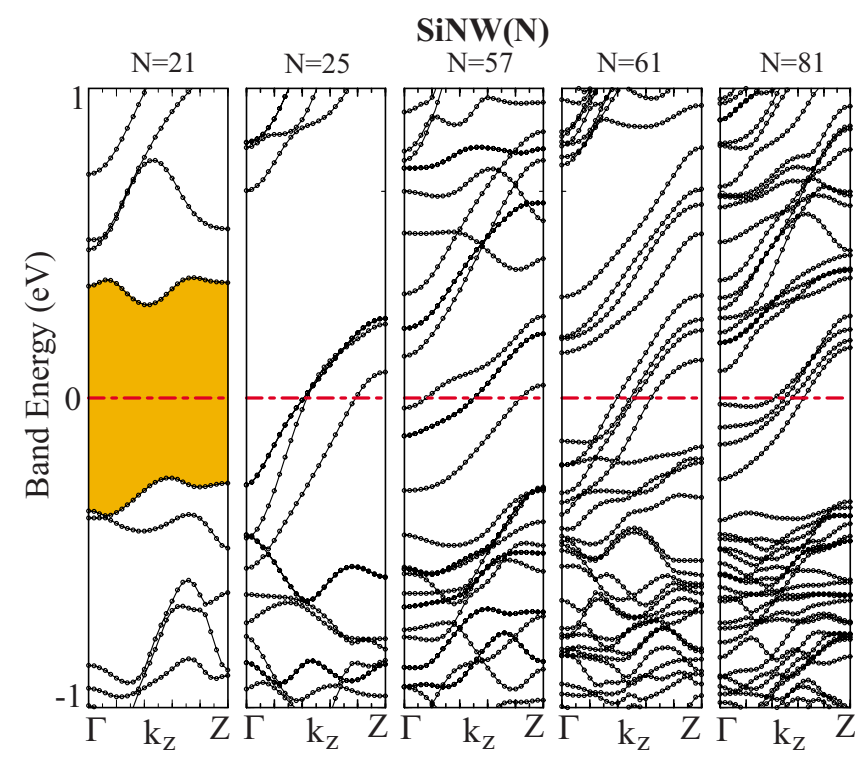

FIG. 6. (Color online) Energy-band structures of structureoptimized bare $\operatorname{SiNW}(N)$ for $N=21,25,57,61$, and 81 . Shaded area is the band gap. Zero of energy is set to Fermi level.

multiple bands crossing the Fermi level, their ballistic quantum conductance given by $G=n h / 2 e$ is high and supports their use as conducting interconnect between molecular or nanoelectronic devices. However, all these nanowires become semiconductor upon the termination of dangling bonds by $\mathrm{H}$ atom.

Because of confinement effects, $E_{G}$ was known to increase with decreasing diameter $D$, displaying the relation $E_{G} \propto 1 / D$. However, our study reveals that $E_{G}$ depends not only on $D$ but also on the geometry of the cross section. In particular, we found that $E_{G}$ of the structure-optimized $\mathrm{H}-\mathrm{SiNW}$ for a given $N$ depends on whether the bare $\mathrm{SiNW}$ is relaxed before it is passivated with hydrogen or not. As an

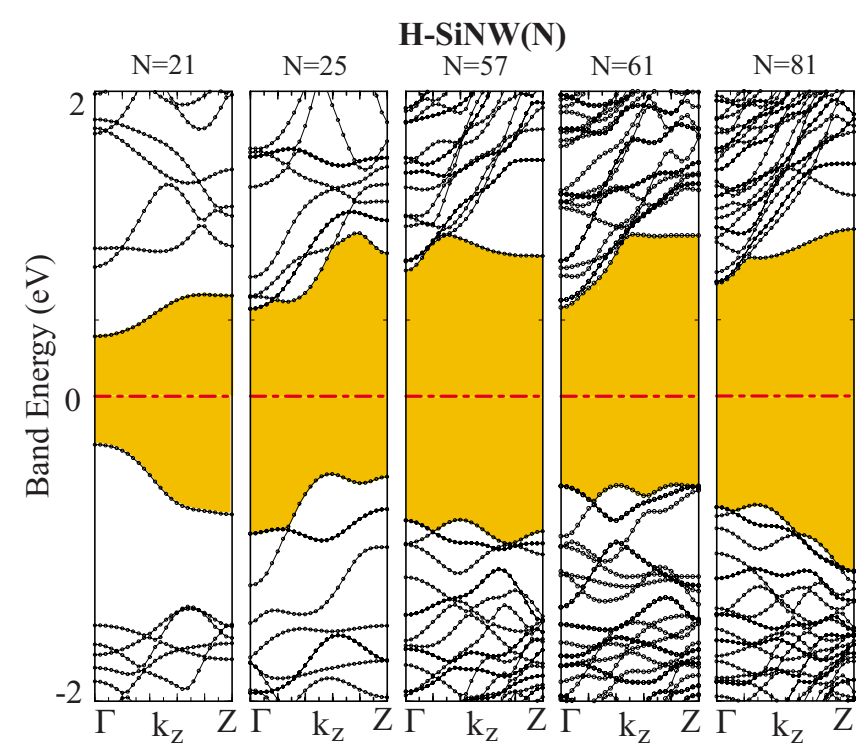

FIG. 7. (Color online) Energy-band structures of optimized $\mathrm{H}-\operatorname{SiNW}(N)$ for $N=21,25,57,61$, and 81 . Shaded area is the band gap. Zero of energy is set to Fermi level. 
TABLE III. The binding energy relative to a free TM atom $E_{B}$, binding energy relative to the TM crystal $E_{B}^{\prime}$, magnetic moment per unit cell $\mu$, and minimum band gap $E_{G}(\mathrm{~S}$ : semiconductor, M: metal, HM: half-metal) of $\mathrm{H}-\mathrm{SiNW}(N)$ covered with $\mathrm{TM}$ at $\Theta=1$ for $N=21,25$, and 57. $\mathrm{TM}=\mathrm{Ti}, \mathrm{Fe}, \mathrm{Mn}, \mathrm{Cr}$, and $\mathrm{Co}$.

\begin{tabular}{|c|c|c|c|c|c|c|c|c|c|c|c|c|}
\hline & \multicolumn{4}{|c|}{$N=21$} & \multicolumn{4}{|c|}{$N=25$} & \multicolumn{4}{|c|}{$N=57$} \\
\hline & $\begin{array}{c}E_{B} \\
(\mathrm{eV})\end{array}$ & $\begin{array}{c}E_{B}^{\prime} \\
(\mathrm{eV})\end{array}$ & $\begin{array}{c}\mu \\
\left(\mu_{B}\right)\end{array}$ & $E_{G}$ & $\begin{array}{c}E_{B} \\
(\mathrm{eV})\end{array}$ & $\begin{array}{c}E_{B}^{\prime} \\
(\mathrm{eV})\end{array}$ & $\begin{array}{c}\mu \\
\left(\mu_{B}\right)\end{array}$ & $E_{G}$ & $\begin{array}{c}E_{B} \\
(\mathrm{eV})\end{array}$ & $\begin{array}{c}E_{B}^{\prime} \\
(\mathrm{eV})\end{array}$ & $\begin{array}{c}\mu \\
\left(\mu_{B}\right)\end{array}$ & $E_{G}$ \\
\hline $\mathrm{Ti}$ & 3.74 & -1.47 & 0 & $S$ & 4.64 & -0.51 & 0 & M & 3.69 & -1.46 & 0 & M \\
\hline $\mathrm{Fe}$ & 3.71 & -1.07 & 2 & S & 3.52 & -1.24 & 2 & $\mathrm{~S}$ & 3.32 & -1.43 & 2 & $\mathrm{~S}$ \\
\hline $\mathrm{Mn}$ & 1.79 & & 3.12 & M & 2.27 & & 3 & HM & 1.82 & & 3 & HM \\
\hline $\mathrm{Cr}$ & 1.81 & -1.80 & 4.41 & M & 2.27 & -1.34 & 4 & HM & 1.88 & -1.73 & 4 & $\mathrm{HM}$ \\
\hline $\mathrm{Co}$ & 4.41 & -0.78 & 1 & HM & 4.12 & -1.07 & 0 & M & 4.08 & -1.06 & 1 & $\mathrm{~S}$ \\
\hline
\end{tabular}

example, we calculated the band gap of $\mathrm{H}-\operatorname{SiNW}(N)-b$ for $N=21,37$, and 57, and found $E_{G}=2.5,2.1$, and $1.7 \mathrm{eV}$, for $N=21,37$, and 57, respectively. This also shows that $E_{G}$ increases significantly if the ideal and bare SiNWs are not relaxed before $\mathrm{H}$ termination. It should be noted that $E_{G}$ for bulk silicon is calculated as $0.65 \mathrm{eV}$ which indicates that H-SiNWs have much wider $E_{G}$ than their crystal counterpart.

The underestimation of the band gap of H-SiNWs is another important issue we want to address. Recent calculations by Zhao et al. ${ }^{52}$ have shown that GW corrections over the local-density approximation (LDA) band gaps is found to be as large as $\sim 1.5 \mathrm{eV}$ for $1 \mathrm{~nm}<D<1.5 \mathrm{~nm}$. Furthermore, GW corrections decrease with increasing $D$, and eventually becomes fixed for large $D$.

\section{FUNCTIONALIZATION BY TRANSITION METAL ATOMS}

\section{A. Energetics of transition metal adsorption}

The electronic properties of bare and hydrogen-terminated SiNWs can be modified through adsorption of TM atoms on the surface of the wire. We considered the adsorption of $3 d$-TM atoms (such as Ti, $\mathrm{Cr}, \mathrm{Mn}, \mathrm{Fe}$, and $\mathrm{Co}$ ) on the surface of bare as well as $\mathrm{H}-\mathrm{SiNW}(N)$ s for $N=21,25$, and 57 . The coverage of TM atoms $\Theta$ is defined as the number $n$ of TM atoms adsorbed per primitive unit cell of the nanowire. Accordingly, one TM atom adsorbed to each primitive unit cell is designated as $\Theta=1$. For SiNWs under study, one can distinguish four to five different adsorption sites. The energetics of TM atom adsorption has been extensively examined for all these sites for $N=21,25$, and 57 .

The binding energy corresponding to $\Theta=1$ is calculated according to the expression $E_{B}=E_{T}[\mathrm{H}-\operatorname{SiNW}(N)]+E_{T}[\mathrm{TM}]$ $-E_{T}[\mathrm{H}-\mathrm{SiNW}(N)+\mathrm{TM}]$, in terms of the total energy of optimized $\mathrm{H}-\mathrm{SiNW}(N)$ and TM adsorbed $\mathrm{H}-\operatorname{SiNW}(N)$ [specified as $\mathrm{H}-\mathrm{SiNW}(N)+\mathrm{TM}]$, and the total energy of a linear chain of TM atoms having the same lattice parameter $c_{0}$ as $\mathrm{H}-\operatorname{SiNW}(N)$, all calculated in the same supercell. Here the total energies, $E_{T}$, are obtained from spin-polarized calculations since TM adsorbed $\mathrm{H}-\mathrm{SiNW}$ normally have magnetic ground state. The calculated energy difference between spinunpolarized and spin-polarized energies, namely, $\Delta E^{m}=E_{T}^{\mathrm{su}}$ $-E_{T}^{\mathrm{sp}}$, is positive. Since the coupling between nearest- neighbor TM atoms has been subtracted through the calculation of $E_{T}[\mathrm{TM}], E_{B}$ can be taken as the binding energy of single isolated TM atom except for the effect of back donation of charge from TM-Si bond to TM-TM bonds. As one expects, the binding energy relative to the bulk TM crystal $E_{B}^{\prime}$ is negative for $\Theta=1$, indicating an endothermic process. However, in the case of high coverage of TM atoms corresponding to $\Theta=n$ with $n>1, \quad E_{B}^{\prime}=\left(E_{T}[\mathrm{H}-\operatorname{SiNW}(N)]\right.$ $\left.+n E_{T}\left[\mathrm{TM}_{\text {bulk }}\right]-E_{T}[\mathrm{H}-\mathrm{SiNW}(N)+n \mathrm{TM}]\right) / n$, can be positive since the coupling of adsorbed TM atoms is included. Calculated results of binding energies, magnetic moments, and band gaps are presented for $N=21,25$, and 57 in Table III for the most energetic adsorption sites. In contrast to usually weak binding of $3 d$-TM atoms on the single-wall carbon nanotubes, the binding energies of TM atoms on $\mathrm{H}-\mathrm{SiNW}$ at $\Theta=1$ are significant and involve charge transfer from TM atom to $\mathrm{Si}$ atoms at close proximity. ${ }^{53}$ The transfer of charge from adsorbed TM atom to the nanowire is estimated by using Mulliken analysis. It is $0.5 e$ from Co to $\mathrm{H}-\mathrm{SiNW}(21)$. The charge transfer from $\mathrm{Cr}$ to $\mathrm{H}-\mathrm{SiNW}(25)$ and $\mathrm{H}-\mathrm{SiNW}(57)$ is even higher, and is calculated to be $0.8 e$ and $0.9 e$, respectively. High charge transfer implies strong chemical interaction.

\section{B. Energy-band structure}

The electronic band structure of TM adsorbed $\mathrm{H}-\operatorname{SiNW}(N)$ s at $\Theta=1$ are shown in Figs. 8-11. We obtain different types of electronic structure depending on the type of adsorbed TM atom and $N$. H-SiNW(21)+ Ti is a nonmagnetic semiconductor but it is nonmagnetic metal for $N=25$ and 57. For $N=21,25$, and $57, \mathrm{H}-\mathrm{SiNW}(N)+\mathrm{Fe}$ is ferromagnetic semiconductor with integer number of spins per primitive cell. It has different band gap for different spin directions. In contrast, $\mathrm{H}-\mathrm{SiNW}(21)+\mathrm{Mn}$ and $\mathrm{H}-\mathrm{SiNW}(21)+\mathrm{Cr}$ are ferromagnetic metals with significant amounts of net magnetic moment per primitive cell.

The situation with $\mathrm{H}-\mathrm{SiNW}(21)+\mathrm{Co}, \mathrm{H}-\mathrm{SiNW}(25)$ $+\mathrm{Mn}(\mathrm{Cr})$, and $\mathrm{H}-\mathrm{SiNW}(57)+\mathrm{Mn}(\mathrm{Cr})$ (Ref. 54) are different from the above cases: these nanowires have half-metallic ground state. Owing to the broken spin degeneracy, energy bands, $E_{n}(\mathbf{k}, \uparrow)$ and $E_{n}(\mathbf{k}, \downarrow)$, split, and the nanowire remains to be an insulator for one spin direction of electrons but becomes conductor for the opposite spin direction. The den- 


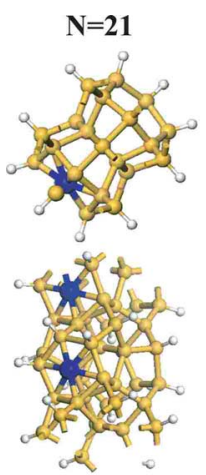

(a)

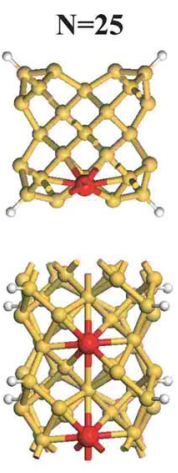

(b)

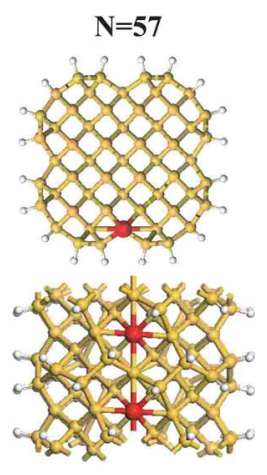

(c)

FIG. 8. (Color online) Top and side views of H-SiNW(21) +Co (first column), (b) $\mathrm{H}-\mathrm{SiNW}(25)+\mathrm{Cr}(\mathrm{Mn})$ (second column), and (c) $\mathrm{H}-\mathrm{SiNW}(57)+\mathrm{Cr}(\mathrm{Mn})$ (third column).

sity of majority- and minority-spin states, namely, $D(E, \uparrow)$ and $D(E, \downarrow)$, display a $100 \%$ spin polarization $P$ at $E_{F}$,

$$
P=\left[D\left(E_{F}, \uparrow\right)-D\left(E_{F}, \downarrow\right)\right] /\left[D\left(E_{F}, \uparrow\right)+D\left(E_{F}, \downarrow\right)\right] .
$$

For the half-metallic nanowires, $\Delta E^{m}$ was calculated to be 0.04, 0.92, and $0.94 \mathrm{eV}$. Comparison of bands of $\mathrm{H}-\operatorname{SiNW}(N)$ in Fig. 7 with those of $\mathrm{H}-\mathrm{SiNW}(N)+\mathrm{TM}$ in Figs. 9-11 reveals that the bands gaps of $\mathrm{H}-\mathrm{SiNW}$ are modified and reduced for one spin direction. On the other hand, two or three bands of the opposite spin states cross $E_{F}$ and attribute a metallicity to the nanowire. These metallic bands are composed of TM-3 $d$ and $\mathrm{Si}-3 p$ hybridized states with higher TM contribution.

Half-metallic state has been a subject of interest since it was predicted first by de Groot et al. ${ }^{37}$ Initial efforts have been devoted to realize half metals using Heusler alloys or

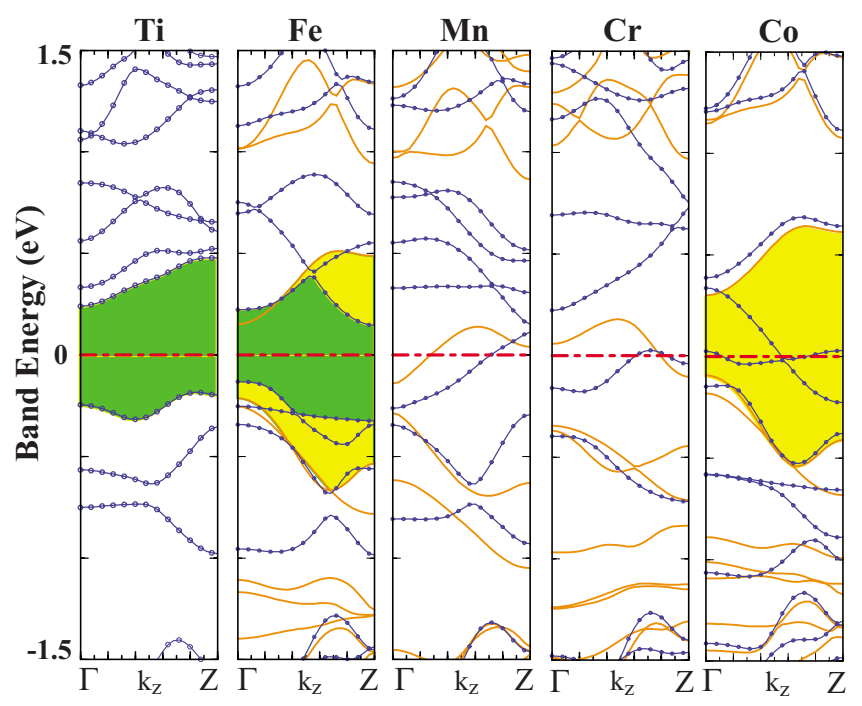

FIG. 9. (Color online) Spin-polarized energy-band structures of $\mathrm{H}-\mathrm{SiNW}(21)$ covered by TM atoms at $\Theta=1$. Band gaps of electrons with different spin directions are shown by different contrast. Bands described by dotted (blue) and continuous (orange) lines are for minority- and majority-spin states, respectively. Zero of energy is set to Fermi level.
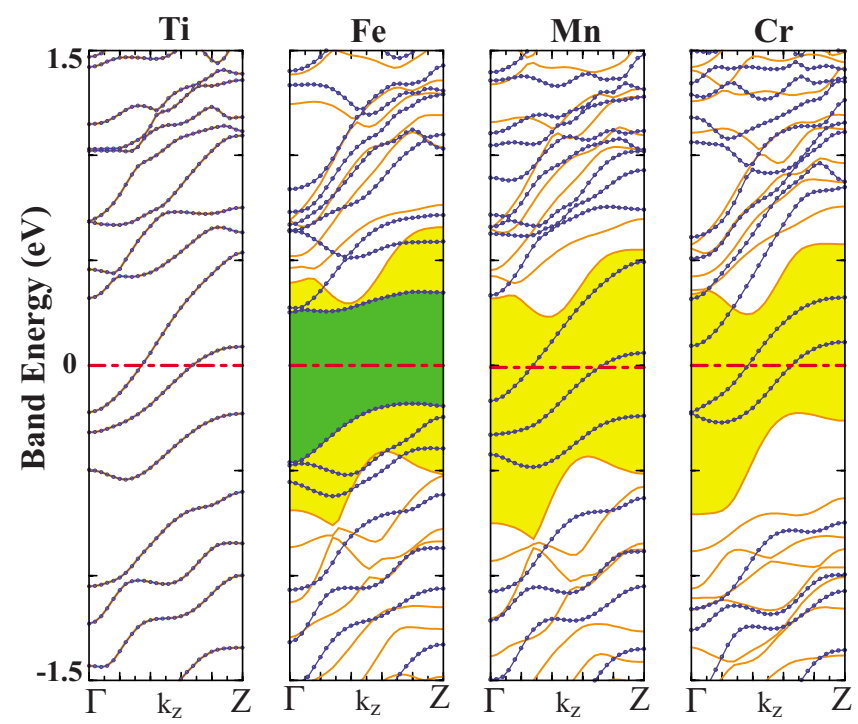

FIG. 10. (Color online) Energy-band structures of H-SiNW(25) covered by TM atoms at $\Theta=1$. Band gaps of electrons with different spin directions are shown by different contrast. Bands described by dotted (blue) and continuous (orange) lines are for minority and majority-spin states, respectively. Zero of energy is set to Fermi level.

TM-doped compound semiconductors in three-dimensional (3D) crystals and in thin films. ${ }^{55,56}$ Qian et al. ${ }^{57}$ have proposed that half-metallic heterostructures can be formed from $\delta$-doped Si crystals. Recently, Son et al. ${ }^{58}$ predicted halfmetallic properties of graphene nanoribbons under bias voltage. Earlier, stable 1D half metals have been also predicted for TM atom-doped armchair single-wall carbon nanotubes ${ }^{59}$ and linear carbon chains. ${ }^{60}$
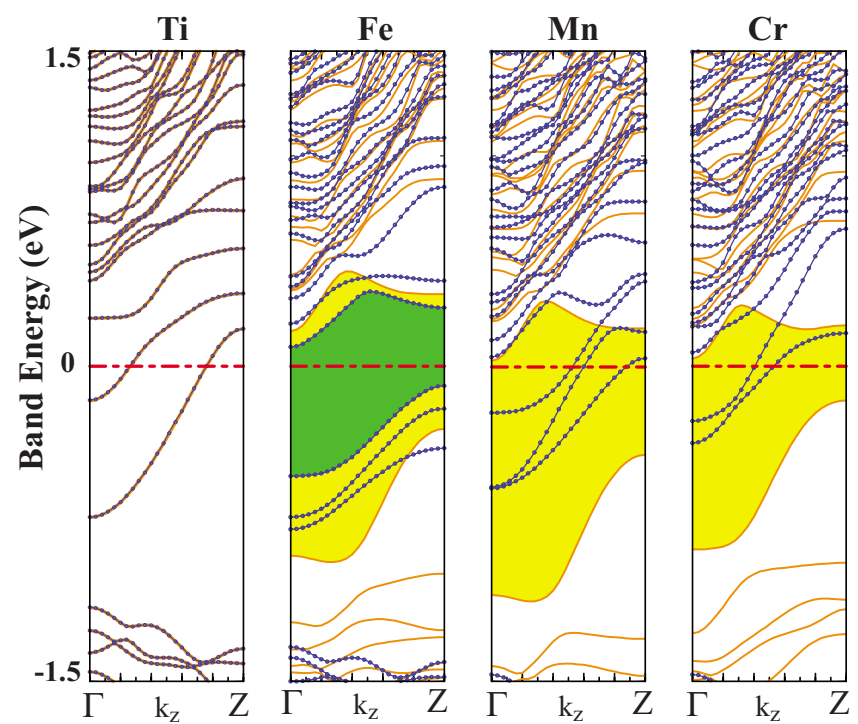

FIG. 11. (Color online) Energy-band structures of H-SiNW(57) covered by TM atoms at $\Theta=1$. Band gaps of electrons with different spin directions are shown by different contrast. Bands described by dotted (blue) and continuous (orange) lines are for minority- and majority-spin states, respectively. Zero of energy is set to Fermi level. 

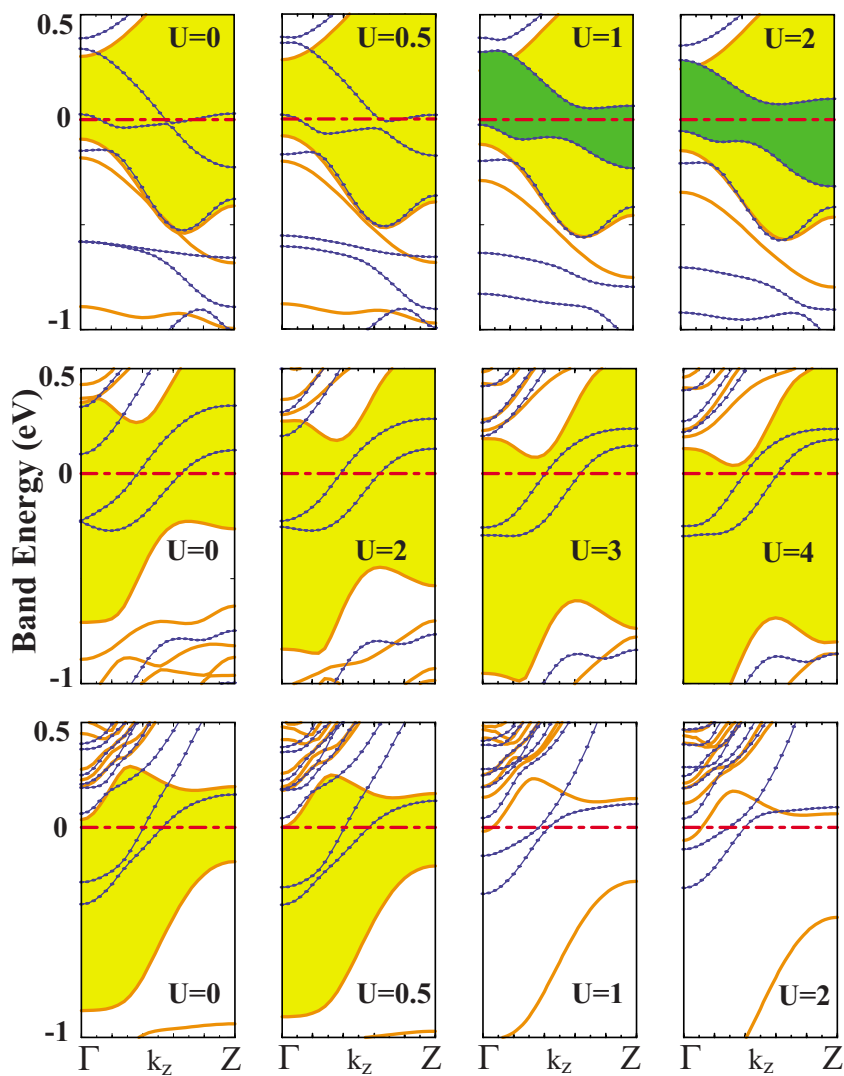

FIG. 12. (Color online) Energy-band structures of $\mathrm{H}-\mathrm{SiNW}(N)$ covered by TM at $\Theta=1$ are calculated by using $\mathrm{LDA}+U$ method. Upper panels: Energy-band structures of $\mathrm{H}-\mathrm{SiNW}(21)+\mathrm{Co}$ with different values of $U$. Middle panels: $\mathrm{H}-\mathrm{SiNW}(25)+\mathrm{Cr}$. Bottom panels: $\mathrm{H}-\mathrm{SiNW}(57)+\mathrm{Cr}$.

As discussed in Sec. III A, we noted that the band-gap values calculated within DFT here are smaller than their actual values. ${ }^{52}$ At this point we address the question whether the half-metallic ground states predicted using DFT is realistic or is an artifact. Since the present calculations predict already a band gap between occupied and unoccupied majority-spin bands, the semiconducting state should be realistic; the actual band gap may be larger than we predicted. As for the metallic minority-spin bands in the gap, they are reminiscent of the linear TM chain having the same lattice constant as H-SiNW $(N)+\mathrm{TM}$. The dispersion of these bands increases in the half-metallic state due to indirect TM-TM interaction via $\mathrm{Si}$ atoms at close proximity of adsorbed TM atom. Briefly, we believe that the present conclusion concerning the half-metallic state is realistic, and underestimated band gap is not affected in any essential manner as long as the optical properties of a H-SiNW+TM is not concerned.

\section{Stability of half-metallic state}

Whether the half-metallic ground state is robust under deformation is another issue we address. We found that under uniaxial compression stress the minimum of the conduction band of majority-spin states rises above $E_{F}$. The minimum of conduction band is, however, lowered under tensile stress to close the band gap. Therefore, the half-metallic state is sen-

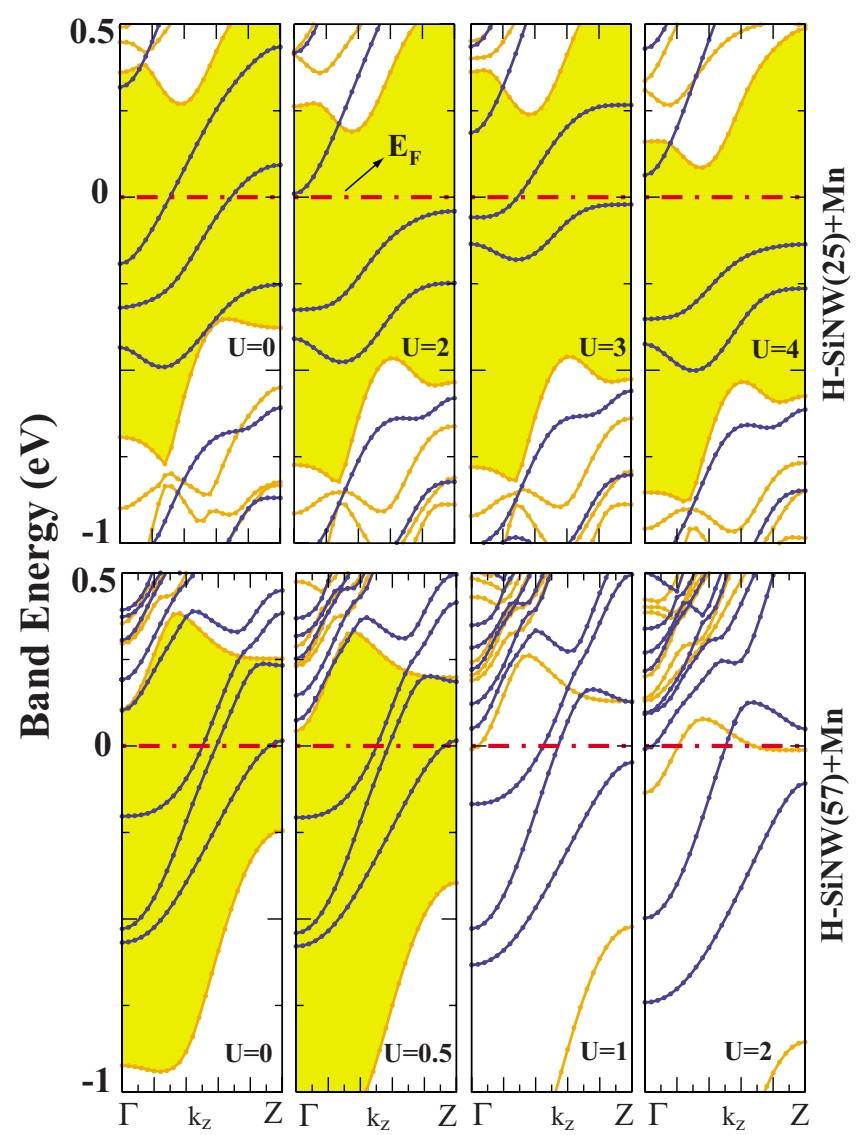

FIG. 13. (Color online) Energy-band structures of $\mathrm{H}-\mathrm{SiNW}(25)+\mathrm{Mn}$ and $\mathrm{H}-\mathrm{SiNW}(57)+\mathrm{Mn}$ calculated by using $\mathrm{LDA}+U$ method.

sitive to the tensile strain for the nanowires that have the conduction-band edge close to $E_{F}$. In our case, since the conduction-band edge of $\mathrm{H}-\mathrm{SiNW}(25)+\mathrm{Cr}(\mathrm{Mn})$ is away from $E_{F}$, their half-metallic state is robust. For $\mathrm{H}-\mathrm{SiNW}(57)+\mathrm{Cr}(\mathrm{Mn})$ having conduction-band edge close to $E_{F}$, their semiconducting state for majority-spin bands may transform to semimetal at high tensile strain.

Since H-SiNW + TM nanowire is one dimension, the metallic minority-spin bands crossing $E_{F}$ are usually prone to Peierls distortion. The form of the bands shown in Figs. 10 and 11 eliminates the possibility of Peierls distortion. However, $\mathrm{H}-\mathrm{SiNW}(21)+\mathrm{Co}$, having metallic bands crossing at $E_{F}$, can become a semiconductor for both spin direction under Peierls distortion.

Another source of instability can be the on-site Coulomb interaction. It has been argued that the spin-dependent GGA may fail to properly represent localized $3 d$ electrons. It may be possible that on-site repulsive Coulomb interaction destroys the half-metallic state. To examine the effect of on-site Coulomb repulsion, we carried out $\mathrm{LDA}+U$ calculations. ${ }^{61}$ The energy-band structures of half-metallic nanowires calculated for different values of $U$ are shown in Figs. 12 and 13. Based on this analysis, while H-SiNW(21) + Co may not be stable and changes to ferromagnetic semiconductor even at $U=0.5, \mathrm{H}-\mathrm{SiNW}(25)+\mathrm{Cr}(\mathrm{Mn})$ can keep their half-metallic state even for $U=4$. As an intermediatory situation, $\mathrm{H}-\mathrm{SiNW}(57)+\mathrm{Cr}(\mathrm{Mn})$ can keep their half-metallic states until $U \sim 1$ 


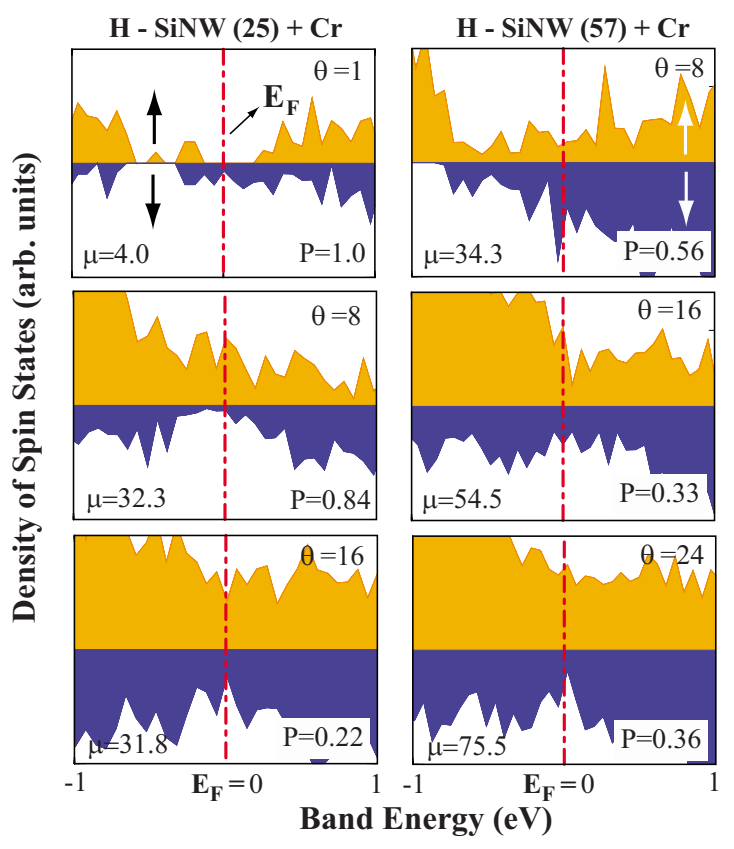

FIG. 14. (Color online) Density of majority- $D(E, \uparrow)$ (dark) and minority- $D(E, \downarrow)$ (light) spin states of $\mathrm{H}-\mathrm{SiNW}(25)+\mathrm{Cr}$ and $\mathrm{H}-\mathrm{SiNW}(57)+\mathrm{Cr}$ for different coverage $\Theta$ of TM atoms (i.e., number of TM atoms per cell). For each case the calculated polarization $P$ and net magnetic moment $\mu$ (in Bohr magnetons per cell) are shown in corresponding panels.

It should be noted that the half-metallic state predicted in this study can occur in periodic and infinite structures. However, the adsorption of TM atoms on a H-SiNW cannot be perfect and periodic. The size of a half-metallic H-SiNW $+\mathrm{TM}$ as a spintronic device has to be finite. As a result, the deviations from the perfect and periodic structures may cause the spin polarization at $E_{F}, P$, to decrease from perfect value $P=1.0$. This means the destruction of ideal half metallicity. Ideal half-metallic state can also be destroyed when $\Theta>1$. Nonetheless, even if $P<1.0$, one can still meet the requirements for specific spintronic devices if the high spin polarization is achieved at $E_{F}$. Here, we examined electronic and magnetic properties of $\mathrm{H}-\mathrm{SiNW}+\mathrm{TM}$ at high TM coverage with the objective of achieving high spin polarization at high $\Theta$.

Figure 14 shows the calculated density of majority- and minority-spin states of $\mathrm{H}-\mathrm{SiNW}(25)$ and $\mathrm{H}-\mathrm{SiNW}(57)$ covered with $\mathrm{Cr}$ for different values of $\Theta$. H-SiNW $(25)+\mathrm{Cr}$, which is a half metal with $\mu=4 \mu_{B}$ and $P=1.0$ at $\Theta=1$, becomes a ferromagnetic metal for both spin directions with $\mu=32.3 \mu_{B}$ and $P=0.84$ at $\Theta=8$. Clearly, the half-metallic state disappeared but due to the high spin polarization, this nanowire can still be used as a spintronic material. Upon increasing $\Theta$ to 16 , spin polarization decreased to $P=0.22$. $E_{F} \mathrm{H}-\mathrm{SiNW}(57)+\mathrm{Cr}$ at $\Theta=8$ has $P=0.56$ and $\mu=34.3 \mu_{B}$. The net magnetic moment per primitive cell increases to $75.5 \mu_{B}$ while $P$ decreases to 0.36 . It appears that $P$ as well as $\mu$ exhibit variations depending on $\Theta, N$, and the type of TM atom. One can achieve high $P$ by covering specific $\mathrm{H}-\mathrm{SiNWs}$ with TM atoms for spintronic applications.

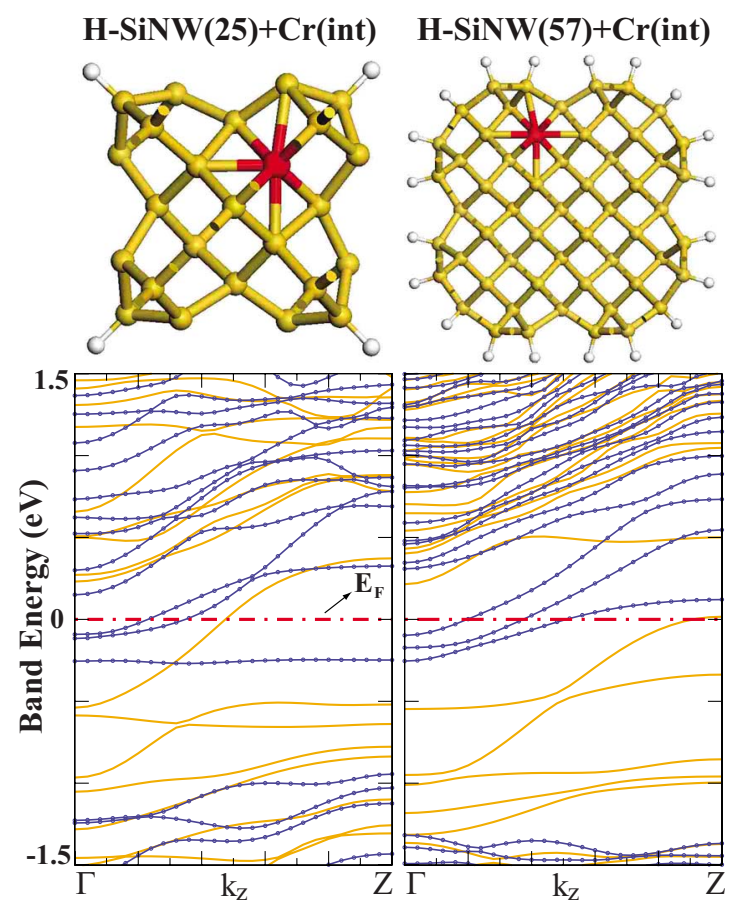

FIG. 15. (Color online) The atomic structures of the cross section of $\mathrm{H}-\mathrm{SiNW}(25)+\mathrm{Cr}$ (int) and $\mathrm{H}-\mathrm{SiNW}(57)+\mathrm{Cr}$ (int), where $\mathrm{Cr}$ is placed at the interstitial sites below the surface. Light (orange) and dotted (blue) bands correspond to the majority- and minorityspin states.

\section{Cr doping at the interstitial site}

Having analyzed the possible adsorption sites of TM atoms on the surface of SiNW and its effects on electronic and magnetic properties, we next consider $\mathrm{Cr}$ atom held at an interstitial site of $\mathrm{H}-\mathrm{SiNW}(25)$ and $\mathrm{H}-\mathrm{SiNW}(57)$ nanowires. Such a situation can be realized when a TM atom can diffuse below the surface at high temperature. One of the possible interstitial sites is expected to be shown in Fig. 15, which is close to a hollow zone. After relaxation it is found that $\mathrm{Cr}$ atom can settle into a cage of seven $\mathrm{Si}$ atoms without deforming $\mathrm{H}-\mathrm{SiNW}(25)$ [H-SiNW(57)]; their total energy is only 10 (16) meV higher than the most energetic configuration obtained for the adsorption of $\mathrm{Cr}$ to their surface but $E_{b}$ is slightly reduced and is calculated as $2.27(1.86) \mathrm{eV}$. We found that the ground state of the system was to be metallic for $\mathrm{H}-\mathrm{SiNW}(25)$ as shown in Fig. 15 with a magnetic moment of $2.93 \mu_{B}$ per cell. The interaction of $\mathrm{Cr}$ with more $\mathrm{Si}$ atoms and hence the hybridization of more $\mathrm{Cr} 3 d$ and $\mathrm{Si} 3 p$ orbitals destroys the HM behavior and makes the wire metallic. When the energy-band structure in Fig. 15 is compared with the external counterpart, it is seen that the effects of interstitial site are more dramatic for $\mathrm{H}-\mathrm{SiNW}(25)$, which has smaller radius. According to the band structure displayed in Fig. 15, $\operatorname{SiNW}(57)+\mathrm{Cr}($ int $)$ appears to be half-metallic except that the highest valence band of majority spins touches the Fermi level at the $Z$ point of the Brillouin zone. Probably, the half-metallic behavior is expected to be secured by GW corrections. The net magnetic moment is calculated to be $3.83 \mu_{B}$ per cell. 


\section{CONCLUSIONS}

We investigated the atomic structure, elastic, electronic, and magnetic properties of small diameter silicon nanowires oriented along [001] direction. We considered bare, hydrogen-passivated, and 3d-TM (Ti, Fe, Mn, Cr, and Co) decorated SiNWs. Here we summarize important predictions of our study: (i) the structure of ideal rods (i.e., SiNWs having ideal bond distances and bond angles) cut from the bulk Si crystal undergo massive reconstruction as a result of structure optimization. (ii) Structure optimized bare SiNWs are generally metallic due to surface dangling bonds. At very small diameters, bare SiNWs can be a semiconductor. (iii) Upon passivation of dangling bonds with hydrogen atoms, the dangling-bond surface states disappear and the metallic nanowire becomes semiconductor with sizable band gap. It has been known that due to confinement effects band gap normally increases with decreasing diameter in the range of 1-2 nm but is stabilized at a constant value for large diameter. It was found that this rule, however, may not be valid if the bare SiNW is relaxed before or after its passivation with hydrogen atoms. (iv) The mechanical properties and hence the strength of bare SiNWs investigated in this paper vary when their surface is passivated with hydrogen atoms. Interestingly, squarelike cross sections are found to be slightly stronger than roundlike cross sections. (v) $3 d$-TM atoms can be adsorbed on specific sites on the surface of hydrogenpassivated SiNWs with significant binding energy, and they attribute magnetic properties. At low coverage of TM atoms,
$\mathrm{H}-\mathrm{SiNW}$ become either ferromagnetic insulator or half metallic depending on the type of adsorbate, as well as the diameter of the nanowire. Half-metallic nanowires are insulators (semiconductors) for one spin direction of electrons but become metal for the opposite direction of spin direction. Further analysis based on $\mathrm{LDA}+U$ calculations shows that half-metallic properties are robust for specific nanowires. (vi) When covered with more TM atoms, the perfect halfmetallic state of $\mathrm{H}-\mathrm{SiNW}$ is disturbed but for certain cases, the spin polarization at $E_{F}$ continues to be high for applications such as spin valves. High magnetic moment obtained at high TM coverage is another remarkable result which may lead to the fabrication of nanomagnets for various applications. (vii) $\mathrm{Cr}$ atoms held at the interstitial sites below the surface give rise to metallic ferromagnetic ground state.

In conclusion, we predict that silicon nanowires can gain a wide range of interesting properties when they are functionalized with TM atoms, such as half metals, onedimensional ferromagnetic semiconductors or ferromagnetic metals, and nanomagnets. Present results hold the promise for the use of silicon nanowires functionalized by $3 d$-transition metal atoms in nanospintronics including magnetoresistance, spin valve, and nonvolatile memories.

\section{ACKNOWLEDGMENT}

This work has been partially supported by TUBITAK under Grant No. TBAG-104T536. *ciraci@fen.bilkent.edu.tr

${ }^{1}$ For a review, see, for example, S. Ciraci, A. Buldum, and I. P. Batra, J. Phys.: Condens. Matter 13, R537 (2001).

${ }^{2}$ E. Durgun, R. T. Senger, H. Mehrez, S. Dag, and S. Ciraci, Europhys. Lett. 73, 642 (2006).

${ }^{3}$ S. Iijima, Nature (London) 354, 56 (1991).

${ }^{4}$ H. Ohnishi, Y. Kondo, and K. Takayanagi, Nature (London) 395, 780 (1998).

${ }^{5}$ H. Dai, E. W. Wong, Y. Z. Lu, S. Fan, and C. M. Lieber, Nature (London) 375, 769 (1995); Y. Zhang and H. Dai, Appl. Phys. Lett. 77, 3015 (2000); Y. Zhang, N. W. Franklin, R. J. Chen, and H. Dai, Chem. Phys. Lett. 331, 35 (2000).

${ }^{6}$ S. Dag, E. Durgun, and S. Ciraci, Phys. Rev. B 69, 121407 (2004).

${ }^{7}$ S. Tongay, R. T. Senger, S. Dag, and S. Ciraci, Phys. Rev. Lett. 93, 136404 (2004); S. Tongay, S. Dag, E. Durgun, R. T. Senger, and S. Ciraci, J. Phys.: Condens. Matter 17, 3823 (2005); R. T. Senger, S. Tongay, S. Dag, E. Durgun, and S. Ciraci, Phys. Rev. B 71, 235406 (2005), and references therein.

${ }^{8}$ P. Sen, O. Gulseren, T. Yildirim, I. P. Batra, and S. Ciraci, Phys. Rev. B 65, 235433 (2002).

${ }^{9}$ E. Durgun, S. Tongay, and S. Ciraci, Phys. Rev. B 72, 075420 (2005).

${ }^{10}$ D. P. Yu, C. S. Lee, I. Bello, X. S. Sun, Y. H. Tang, G. W. Zhou, Z. G. Bai, Z. Zhang, and S. Q. Feng, Solid State Commun. 105, 403 (1998).

${ }^{11}$ J. Hu, T. W. Odom, and C. M. Lieber, Acc. Chem. Res. 32, 435
(1999).

${ }^{12}$ K. Hiruma, M. Yazawa, T. Katsuyama, K. Ogawa, K. Haraguchi, M. Koguchi, and H. Kakibayashi, J. Appl. Phys. 77, 447 (1995).

${ }^{13}$ W. Q. Han, S. S. Fan, Q. Q. Li, and Y. D. Hu, Science 277, 1287 (1997).

${ }^{14}$ C. R. Martin, Science 266, 1961 (1994).

${ }^{15}$ Y. F. Zhang, L. S. Liao, W. H. Chan, S. T. Lee, R. Sammynaiken, and T. K. Sham, Phys. Rev. B 61, 8298 (2000).

${ }^{16}$ D. D. D. Ma, C. S. Lee, F. C. K. Au, S. Y. Tong, and S. T. Lee, Science 299, 1874 (2003).

${ }^{17}$ M. Menon and E. Richter, Phys. Rev. Lett. 83, 792 (1999).

${ }^{18}$ B. Marsen and K. Sattler, Phys. Rev. B 60, 11593 (1999).

${ }^{19}$ Y. Zhao and B. I. Yakobson, Phys. Rev. Lett. 91, 035501 (2003).

${ }^{20}$ J. D. Holmes, K. P. Johnston, R. C. Doty, and B. A. Korgel, Science 287, 1471 (2000).

${ }^{21}$ Y. Wu, Y. Cui, L. Huynh, C. J. Barrelet, D. C. Bell, and C. M. Lieber, Nano Lett. 4, 433 (2004).

${ }^{22}$ R. Rurali and N. Lorente, Phys. Rev. Lett. 94, 026805 (2005).

${ }^{23}$ R. Q. Zhang, Y. Lifshitz, D. D. D. Ma, Y. L. Zhao, Th. Frauenheim, S. T. Lee, and S. Y. Tong, J. Chem. Phys. 123, 144703 (2005).

${ }^{24}$ P. W. Leu, B. Shan, and K. Cho, Phys. Rev. B 73, 195320 (2006).

${ }^{25}$ A. K. Singh, V. Kumar, R. Note, and Y. Kawazoe, Nano Lett. 6, 920 (2006).

${ }^{26}$ Numerous theoretical studies on SiNW have been published in recent years. See, for example: Q. Wang, Q. Sun, and P. Jena, 
Phys. Rev. Lett. 95, 167202 (2005); Nano Lett. 5, 1587 (2005).

${ }^{27}$ Y. Cui, Z. Zhong, D. Wang, W. U. Wang, and C. M. Lieber, Nano Lett. 3, 149 (2003).

${ }^{28}$ Y. Huang, X. Duan, and C. M. Lieber, Small 1, 142 (2005).

${ }^{29}$ X. Duan, Y. Huang, R. Agarwal, and C. M. Lieber, Nature (London) 421, 241 (2003).

${ }^{30}$ M. V. Fernandez-Serra, Ch. Adessi, and X. Blase, Phys. Rev. Lett. 96, 166805 (2006).

${ }^{31}$ E. Durgun, N. Akman, C. Ataca, and S. Ciraci, Phys. Rev. B 76, 245323 (2007).

${ }^{32}$ Y. Cui, Q. Wei, H. Park, and C. M. Lieber, Science 293, 1289 (2001).

${ }^{33}$ X. T. Zhou, J. Q. Hu, C. P. Li, D. D. D. Ma, C. S. Lee, and S. T. Lee, Chem. Phys. Lett. 369, 220 (2003).

${ }^{34}$ J. Hahm and C. M. Lieber, Nano Lett. 4, 51 (2004).

${ }^{35}$ N. Akman, E. Durgun, S. Cahangirov, and S. Ciraci, Phys. Rev. B 76, 245427 (2007).

${ }^{36}$ E. Durgun, D. Cakir, N. Akman, and S. Ciraci, Phys. Rev. Lett. 99, 256806 (2007)

${ }^{37}$ R. A. de Groot, F. M. Mueller, P. G. van Engen, and K. H. J. Buschow, Phys. Rev. Lett. 50, 2024 (1983).

${ }^{38}$ W. E. Pickett and J. S. Moodera, Phys. Today 54(5), 39 (2001).

${ }^{39}$ H. W. Wu, C. J. Tsai, and L. J. Chen, Appl. Phys. Lett. 90, 043121 (2007).

${ }^{40}$ M. C. Payne, M. P. Teter, D. C. Allan, T. A. Arias, and J. D. Joannopoulos, Rev. Mod. Phys. 64, 1045 (1992).

${ }^{41}$ Numerical computations have been carried out by using VASP software: G. Kresse and J. Hafner, Phys. Rev. B 47, 558 (1993); G. Kresse and J. Furthmuller, ibid. 54, 11169 (1996).

${ }^{42}$ Charge transfer, orbital hybridization, and local magnetic moments have been obtained from SIESTA code using local basis set: P. Ordejon, E. Artacho, and J. M. Soler, Phys. Rev. B 53, R10441 (1996).

${ }^{43}$ W. Kohn and L. J. Sham, Phys. Rev. 140, A1133 (1965); P. Hohenberg and W. Kohn, ibid. 136, B864 (1964).

${ }^{44}$ D. Vanderbilt, Phys. Rev. B 41, 7892 (1990).
${ }^{45}$ P. E. Blochl, Phys. Rev. B 50, 17953 (1994).

${ }^{46}$ J. P. Perdew, J. A. Chevary, S. H. Vosko, K. A. Jackson, M. R. Pederson, D. J. Singh, and C. Fiolhais, Phys. Rev. B 46, 6671 (1992).

${ }^{47}$ M. Methfessel and A. T. Paxton, Phys. Rev. B 40, 3616 (1989).

${ }^{48}$ H. J. Monkhorst and J. D. Pack, Phys. Rev. B 13, 5188 (1976).

${ }^{49}$ S. Ismail-Beigi and T. Arias, Phys. Rev. B 57, 11923 (1998).

${ }^{50}$ J. X. Cao, X. G. Gong, J. X. Zhong, and R. Q. Wu, Phys. Rev. Lett. 97, 136105 (2006).

${ }^{51}$ R. Rurali, A. Poissier, and N. Lorente, Phys. Rev. B 74, 165324 (2006).

${ }^{52}$ X. Zhao, C. M. Wei, L. Yang, and M. Y. Chou, Phys. Rev. Lett. 92, 236805 (2004).

${ }^{53}$ E. Durgun, S. Dag, V. M. K. Bagci, O. Gulseren, T. Yildirim, and S. Ciraci, Phys. Rev. B 67, 201401(R) (2003); J. Phys. Chem. B 108, 575 (2004).

${ }^{54}$ The obtained results are also checked for H-SiNW(45) which has different facets (more round cross section), even though it is not more energetic than other types discussed in the paper. $\mathrm{Cr}(\mathrm{Mn})$ adsorption also makes H-SiNW(45) half-metallic with a similar band structure to $\mathrm{H}-\mathrm{SiNW}(57)+\mathrm{Cr}(\mathrm{Mn})$.

${ }^{55}$ J.-H. Park, E. Vescovo, H.-J. Kim, C. Kwon, R. Ramesh, and T. Venkatesan, Nature (London) 392, 794 (1998).

${ }^{56}$ H. Akinaga, T. Manago, and M. Shirai, Jpn. J. Appl. Phys., Part 2 39, L1118 (2000).

${ }^{57}$ M. C. Qian, C. Y. Fong, K. Liu, W. E. Pickett, J. E. Pask, and L. H. Yang, Phys. Rev. Lett. 96, 027211 (2006).

${ }^{58}$ Y.-W. Son, M. L. Cohen, and S. G. Louie, Nature (London) 444, 347 (2006).

${ }^{59}$ C.-K. Yang, J. Zhao, and J. P. Lu, Nano Lett. 4, 561 (2004); Y. Yagi, T. M. Briere, M. H. F. Sluiter, V. Kumar, A. A. Farajian, and Y. Kawazoe, Phys. Rev. B 69, 075414 (2004).

${ }^{60}$ S. Dag, S. Tongay, T. Yildirim, E. Durgun, R. T. Senger, C. Y. Fong, and S. Ciraci, Phys. Rev. B 72, 155444 (2005).

${ }^{61}$ S. L. Dudarev, G. A. Botton, S. Y. Savrasov, C. J. Humphreys, and A. P. Sutton, Phys. Rev. B 57, 1505 (1998). 